\title{
Two-Loop Correction to the Higgs Boson Mass in the MRSSM
}

\author{
Philip Diessner, ${ }^{1}$ Jan Kalinowski, ${ }^{2}$ Wojciech Kotlarski, ${ }^{1,2}$ and Dominik Stöckinger ${ }^{1}$ \\ ${ }^{1}$ Institut für Kern- und Teilchenphysik, TU Dresden, 01069 Dresden, Germany \\ ${ }^{2}$ Faculty of Physics, University of Warsaw, Pasteura 5, 02093 Warsaw, Poland \\ Correspondence should be addressed to Wojciech Kotlarski; wojciech.kotlarski@fuw.edu.pl
}

Received 24 April 2015; Accepted 23 June 2015

Academic Editor: Mark D. Goodsell

Copyright (C) 2015 Philip Diessner et al. This is an open access article distributed under the Creative Commons Attribution License, which permits unrestricted use, distribution, and reproduction in any medium, provided the original work is properly cited. The publication of this article was funded by SCOAP ${ }^{3}$.

\begin{abstract}
We present the impact of two-loop corrections on the mass of the lightest Higgs boson in the minimal $R$-symmetric supersymmetric standard model (MRSSM). These shift the Higgs boson mass up by typically $5 \mathrm{GeV}$ or more. The dominant corrections arise from strong interactions, and from the gluon and its $N=2$ superpartners, the sgluon and Dirac gluino, and these corrections further increase with large Dirac gluino mass. The two-loop contributions governed purely by Yukawa couplings and the MRSSM $\lambda, \Lambda$ parameters are smaller. We also update our earlier analysis which showed that the MRSSM can accommodate the measured Higgs and $W$ boson masses. Including the two-loop corrections increases the parameter space where the theory prediction agrees with the measurement.
\end{abstract}

\section{Introduction}

The recent discovery at the LHC of a particle consistent with the long sought Higgs boson seemingly completes the Standard Model (SM). The mass of the particle is measured with an astonishingly high accuracy of $m_{H}=125.09 \pm$ $0.24 \mathrm{GeV}$ [1]. The precise determination of this mass is of paramount importance not only within the context of the Standard Model, but also for finding the path beyond it. In fact, a number of experimental observations suggest that the SM cannot be the ultimate theory and many theoretical scenarios for the beyond SM (BSM) physics have been proposed in past decades. In some models of BSM, in particular in supersymmetric extensions of the SM, the Higgs boson mass can be predicted. However, the current experimental accuracy is far better than theoretical predictions for Higgs boson mass in any given model of BSM physics. From the point of view of theory, the best accuracy has been achieved in the minimal supersymmetric extension of the SM (MSSM), in which the discovery of the Higgs boson and the determination of its mass have given a new impetus to the theoretical efforts. The most recent improvements comprise the inclusion of leading three-loop corrections [2,3], resummation of leading logarithms beyond the two-loop level $[4,5]$, inclusion of the external momenta of two-loop self-energies $[6,7]$, and the evaluation of the $\mathcal{O}\left(\alpha_{t}^{2}\right)$ contributions in the complex MSSM $[8,9]$. The MSSM twoloop corrections controlled by Yukawa couplings and $\alpha_{s}$ have been known for quite some time for the real MSSM (see the above references for an overview of the literature).

The absence of any direct signal of supersymmetric particle production at the LHC and the observed Higgs boson mass of $\sim 125 \mathrm{GeV}$ being rather close to the upper value of $\sim 135 \mathrm{GeV}$ achievable in the MSSM are a strong motivation to consider nonminimal SUSY scenarios. In fact, nonminimal SUSY models can lift the Higgs boson mass (at the tree-level by new $F$ - or $D$-term contributions or at the loop level from additional new states), which makes these models more natural by reducing fine-tuning. They can also weaken SUSY limits either by predicting compressed spectra or by reducing the expected missing transverse energy or by reducing production cross sections. The comparison of the measured Higgs boson mass with the theoretically predicted values in any given model is therefore highly desirable. Although the theoretical calculations for the SM-like Higgs boson mass in such models are less advanced, progress is being made in the development of highly automated tools which greatly facilitate the computations in nonminimal SUSY models: SARAH [10-12] automatically generates spectrum generators similar to SPheno $[13,14]$; FlexibleSUSY 
[15] automatically generates spectrum generators similar to Softsusy [16].

In a recent paper [17] we considered the MRSSM, a highly motivated supersymmetric model with continuous $R$ symmetry $[18,19]$ distinct from the MSSM. Since $R$-symmetry forbids soft Majorana gaugino masses as well as the higgsino mass term, additional superfields are needed. The MRSSM has been constructed in [20] as a minimal viable model of this type. It contains adjoint chiral superfields with $R$-charge 0 for each gauge sector and two additional Higgs weak iso-doublet superfields with $R$-charge 2 . It has been also argued that $R$-symmetry generically forbids large contributions to CP- and flavor-violating observables due to the absence of chirality-changing Dirac gluino couplings [20, 21], relaxing flavor constraints on the sfermion sector, although recently it has been shown that the dramatic chirality-flip suppression of [20] can only work in a limited number of scenarios, and in general a certain correlation between flavor structure of fermion masses and superpartner spectrum is required [22]. Also, Dirac gluinos suppress the production cross section for squarks, making squarks below the $\mathrm{TeV}$ scale generically compatible with LHC data. Furthermore, models with $R$-symmetry and/or Dirac gauginos contain promising dark matter candidates [23-25], and the collider physics of the extra, non-MSSM-like states has been studied [26-34].

In [17] the complete next-to-leading order computation and discussion of the lightest Higgs boson and $W$ boson masses have been performed. We showed that the model can accommodate measured values of these observables for interesting regions of parameter space with stop masses of order $1 \mathrm{TeV}$ (a similar analysis has been done in [35], where also a welcome reduction of the level of fine-tuning was found). The outcome of the paper was not obvious since in the MRSSM (i) the lightest Higgs boson tree-level mass is typically reduced compared to the MSSM due to mixing with additional scalars, (ii) the stop mixing is absent, and (iii) $R$-symmetry necessarily introduces an $S U(2)$ scalar triplet, which can increase $m_{W}$ already at the tree-level. Nevertheless, we identified benchmark points BMP1, BMP2, and BMP3 illustrating different viable parameter regions for $\tan \beta=$ $3,10,40$, respectively, and also verified that they are not excluded by further experimental constraints from Higgs observables, collider, and low-energy physics.

These promising results motivate a more precise computation of the Higgs boson mass in the MRSSM and a more precise parameter analysis. Technically, this is facilitated by the Mathematica package SARAH, recently updated by providing SPheno routines, which calculate two-loop corrections to the CP-even Higgs scalars masses in the effective potential approximation and the gaugeless limit [36]. This is the level of precision of the established MSSM predictions except for the refinements mentioned above. It is also the level of precision at which the proof [37] applies that the employed regularization by dimensional reduction preserves supersymmetry. First applications of the improved SARAH version to the calculations of the Higgs boson masses in the $R$-parity violating MSSM [38] and next-to-minimal SSM [39] have been published.
Since a judicious choice of the model parameters was needed to meet experimental constraints and an estimate of unknown two-loop contributions was presented, it is of immediate interest to verify our findings at higher precision with the new SARAH version. The aim of the current paper is to calculate two-loop corrections for the Higgs boson mass in the same MRSSM setup as in [17] and present an update of the results obtained there.

The paper is organized as follows. After a short recapitulation of the MRSSM setup in Section 2, we explain in Section 3 our calculation framework and discuss the dependence of two-loop corrections on parameters that entered already at the one-loop level. The dependence on parameters that enter only at the two-loop level is investigated in Section 4. In Section 5 we provide an update to the analysis presented in [17] using the two-loop corrected masses of Higgses, before concluding in Section 6.

\section{The MRSSM}

The MRSSM has been constructed in [20] as a minimal supersymmetric model with unbroken continuous $R$-symmetry. The superpotential of the model reads as

$$
\begin{aligned}
W= & \mu_{d} \widehat{R}_{d} \cdot \widehat{H}_{d}+\mu_{u} \widehat{R}_{u} \cdot \widehat{H}_{u}+\Lambda_{d} \widehat{R}_{d} \cdot \widehat{T} \widehat{H}_{d}+\Lambda_{u} \widehat{R}_{u} \\
& \cdot \widehat{T} \widehat{H}_{u}+\lambda_{d} \widehat{S} \widehat{R}_{d} \cdot \widehat{H}_{d}+\lambda_{u} \widehat{S} \widehat{R}_{u} \cdot \widehat{H}_{u}-Y_{d} \widehat{d} \widehat{q} \cdot \widehat{H}_{d} \\
& -Y_{e} \widehat{e} \widehat{e} \cdot \widehat{H}_{d}+Y_{u} \widehat{u} \hat{q} \cdot \widehat{H}_{u},
\end{aligned}
$$

where $\widehat{H}_{u, d}$ are the MSSM-like Higgs weak iso-doublets and $\widehat{S}, \widehat{T}, \widehat{R}_{u, d}$ are the singlet, weak iso-triplet, and $\widehat{R}$-Higgs weak iso-doublets, respectively. The usual MSSM $\mu$-term is forbidden; instead $\mu_{u, d}$-terms involving $R$-Higgs fields are allowed. $\Lambda, \lambda$-terms are similar to the usual Yukawa terms, where $\widehat{R}$-Higgs and $\widehat{S}$ or $\widehat{T}$ play the role of the quark/lepton doublets and singlets.

The usual soft mass terms of the MSSM scalar fields are allowed just like in the MSSM. In contrast, $A$-terms and soft Majorana gaugino masses are forbidden by $R$-symmetry. The fermionic components of the chiral adjoint, $\widehat{\Phi}_{i}=\widehat{\mathcal{O}}, \widehat{T}, \widehat{S}$ for each standard model gauge group $i=S U(3), S U(2)$, $U(1)$, respectively, are paired with standard gauginos $\widetilde{g}, \widetilde{W}, \widetilde{B}$ to build Dirac fermions and the corresponding mass terms. The Dirac gaugino masses generated by $D$-type spurions produce additional terms with the auxiliary $\mathscr{D}$-fields in the Lagrangian,

$$
\begin{aligned}
V_{D}= & M_{B}^{D}\left(\widetilde{B} \widetilde{S}-\sqrt{2} \mathscr{D}_{B} S\right)+M_{W}^{D}\left(\widetilde{W}^{a} \widetilde{T}^{a}-\sqrt{2} \mathscr{D}_{W}^{a} T^{a}\right) \\
& +M_{O}^{D}\left(\widetilde{g}^{a} \widetilde{O}^{a}-\sqrt{2} \mathscr{D}_{g}^{a} O^{a}\right)+\text { h.c. }
\end{aligned}
$$

which after being eliminated through their equations of motion lead to the appearance of Dirac masses in the scalar sector as well. For our phenomenological studies of two-loop 
effects we take the soft-breaking scalar mass terms that have been considered in [17]

$$
\begin{aligned}
V_{\mathrm{SB}}^{\mathrm{EW}}= & m_{H_{d}}^{2}\left(\left|H_{d}^{0}\right|^{2}+\left|H_{d}^{-}\right|^{2}\right)+m_{H_{u}}^{2}\left(\left|H_{u}^{0}\right|^{2}+\left|H_{u}^{+}\right|^{2}\right) \\
& +\left[B_{\mu}\left(H_{d}^{-} H_{u}^{+}-H_{d}^{0} H_{u}^{0}\right)+\text { h.c. }\right] \\
& +m_{R_{d}}^{2}\left(\left|R_{d}^{0}\right|^{2}+\left|R_{d}^{+}\right|^{2}\right)+m_{R_{u}}^{2}\left|R_{u}^{0}\right|^{2}+m_{R_{u}}^{2}\left|R_{d}^{-}\right|^{2} \\
& +m_{S}^{2}|S|^{2}+m_{T}^{2}\left|T^{0}\right|^{2}+m_{T}^{2}\left|T^{-}\right|^{2}+m_{T}^{2}\left|T^{+}\right|^{2} \\
& +m_{O}^{2}|O|^{2}+\widetilde{d}_{L, i}^{*} m_{q, i j}^{2} \widetilde{d}_{L, j}+\widetilde{d}_{R, i}^{*} m_{d, i j}^{2} \widetilde{d}_{R, j} \\
& +\widetilde{u}_{L, i}^{*} m_{q, i j}^{2} \widetilde{u}_{L, j}+\widetilde{u}_{R, i}^{*} m_{u, i j}^{2} \widetilde{u}_{R, j}+\widetilde{e}_{L, i}^{*} m_{l, i j}^{2} \widetilde{e}_{L, j} \\
& +\widetilde{e}_{R, i}^{*} m_{e, i j}^{2} \widetilde{e}_{R, j}+\widetilde{v}_{L, i}^{*} m_{l, i j}^{2} \widetilde{v}_{L, j},
\end{aligned}
$$

where the holomorphic mass terms for adjoint scalars, which might lead to tachyonic states, have been neglected (see also $[40,41]$ for discussions that these terms can be subdominant within a broad definition of gauge mediation).

The electroweak symmetry breaking (EWSB) is triggered by nonzero vacuum expectation values of $R=0$ neutral EW scalars, which are parameterized as

$$
\begin{aligned}
H_{d}^{0} & =\frac{1}{\sqrt{2}}\left(v_{d}+\phi_{d}+i \sigma_{d}\right), \\
H_{u}^{0} & =\frac{1}{\sqrt{2}}\left(v_{u}+\phi_{u}+i \sigma_{u}\right), \\
T^{0} & =\frac{1}{\sqrt{2}}\left(v_{T}+\phi_{T}+i \sigma_{T}\right), \\
S & =\frac{1}{\sqrt{2}}\left(v_{S}+\phi_{S}+i \sigma_{S}\right) ;
\end{aligned}
$$

$R$-Higgs bosons carry $R$-charge 2 and therefore do not develop vacuum expectation values. We stress that in general the mixing of $\phi_{T}, \phi_{S}$ with $\phi_{u}$ and $\phi_{d}$ leads to a reduction of the lightest Higgs boson mass at the tree-level compared to the MSSM.

\section{Higgs Mass Dependence on $\lambda, \Lambda$ Superpotential Parameters}

We now present the MRSSM Higgs boson mass prediction at the two-loop level. We use the same renormalization scheme as in [17], where all SUSY parameters are defined in the $\overline{\mathrm{DR}}$ scheme and $m_{H_{d}}^{2}, m_{H_{u}}^{2}, v_{S}$, and $v_{T}$ are determined by minimizing the effective potential at the two-loop order. The discussion is divided into two parts. In the present section we begin with the one-loop contributions, which are dominated by terms of $\mathcal{O}\left(\alpha_{t, b, \lambda}\right)$, where $\alpha_{\lambda}$ collectively denotes squares of the superpotential couplings $\lambda_{u, d}$ and $\Lambda_{u, d}$. We then discuss the two-loop contributions of $\mathcal{O}\left(\alpha_{t, b, \lambda}^{2}\right)$, that is, ones which depend on parameters which already play a role at the oneloop level. In the subsequent section we then discuss those two-loop corrections which involve new parameters.
In the usual MSSM, the one-loop contributions to the Higgs boson mass are dominated by top/stop contributions. In the MRSSM, these contributions are also important, but they are simpler since stop mixing is forbidden by $R$ symmetry (corresponding to the MSSM parameter $X_{t} \equiv A_{t}-$ $\mu / \tan \beta=0$ ). This implies that the top/stop contributions cannot reach values as high as in the MSSM for a given stop mass scale. However, as mentioned above, the MRSSM superpotential contains new terms governed by $\lambda_{u, d}$ and $\Lambda_{u, d}$ which have a Yukawa-like structure. References [17, 35] have given a useful analytical approximation for these contributions. In the limit $\lambda=\lambda_{u}=-\lambda_{d}, \Lambda=\Lambda_{u}=\Lambda_{d}$, $v_{S} \approx v_{T} \approx 0$, and large $\tan \beta$, we get

$$
\begin{aligned}
& \Delta m_{H_{1}, \text { eff.pot }, \lambda}^{2}=\frac{2 v^{2}}{16 \pi^{2}}\left[\frac{\Lambda^{2} \lambda^{2}}{2}\right. \\
& +\frac{4 \lambda^{4}+4 \lambda^{2} \Lambda^{2}+5 \Lambda^{4}}{8} \log \frac{m_{R_{u}}^{2}}{Q^{2}} \\
& +\left(\frac{\lambda^{4}}{2}-\frac{\lambda^{2} \Lambda^{2}}{2} \frac{m_{S}^{2}}{m_{T}^{2}-m_{S}^{2}}\right) \log \frac{m_{S}^{2}}{Q^{2}} \\
& +\left(\frac{5}{8} \Lambda^{4}+\frac{\lambda^{2} \Lambda^{2}}{2} \frac{m_{T}^{2}}{m_{T}^{2}-m_{S}^{2}}\right) \log \frac{m_{T}^{2}}{Q^{2}} \\
& -\left(\frac{5}{4} \Lambda^{4}-\lambda^{2} \Lambda^{2} \frac{\left(M_{W}^{D}\right)^{2}}{\left(M_{B}^{D}\right)^{2}-\left(M_{W}^{D}\right)^{2}}\right) \log \frac{\left(M_{W}^{D}\right)^{2}}{Q^{2}} \\
& \left.-\left(\lambda^{4}+\lambda^{2} \Lambda^{2} \frac{\left(M_{B}^{D}\right)^{2}}{\left(M_{B}^{D}\right)^{2}-\left(M_{W}^{D}\right)^{2}}\right) \log \frac{\left(M_{B}^{D}\right)^{2}}{Q^{2}}\right] .
\end{aligned}
$$

This result shows a behavior proportional to $\lambda^{4}, \Lambda^{4}$, and $\log m_{\text {soft }}^{2}$. This is similar to the top/stop contributions as $\lambda$ 's and $Y_{t}$ appear in a similar fashion in superpotential.

We expect therefore that the two-loop result will depend on these model parameters (which already entered at the oneloop level) in a manner similar to the pure top quark/squarks two-loop contributions, that is, similar to the MSSM $\mathcal{O}\left(\alpha_{t}^{2}\right)$ contributions without stop mixing.

In Figures 1 and 2 the dependence of the lightest Higgs boson mass calculated at tree-, one-, and two-loop levels for two benchmarks BMP1 and BMP3 on different model parameters is shown. All parameters except the ones shown on the horizontal axes are set to the values of the benchmark points defined in [17] (see Table 2). Indeed $\lambda, \Lambda$ behavior of the two-loop corrections is very similar to the one of the corresponding one-loop corrections. The numerical impact of the two-loop $\lambda, \Lambda$-contributions is rather small, typically less than $1 \mathrm{GeV}$, except for very large $\left|\lambda_{u}\right|,\left|\Lambda_{u}\right|>1$, where they can reach several GeV. Particularly, the strong $\lambda_{u}$ dependence for large $\lambda_{u}$ is already manifest for the tree-level mass; this is due to the mixing with the singlet state already present in the tree-level mass matrix.

One should remember that very large one-loop contributions are required to bring the predicted Higgs boson mass 

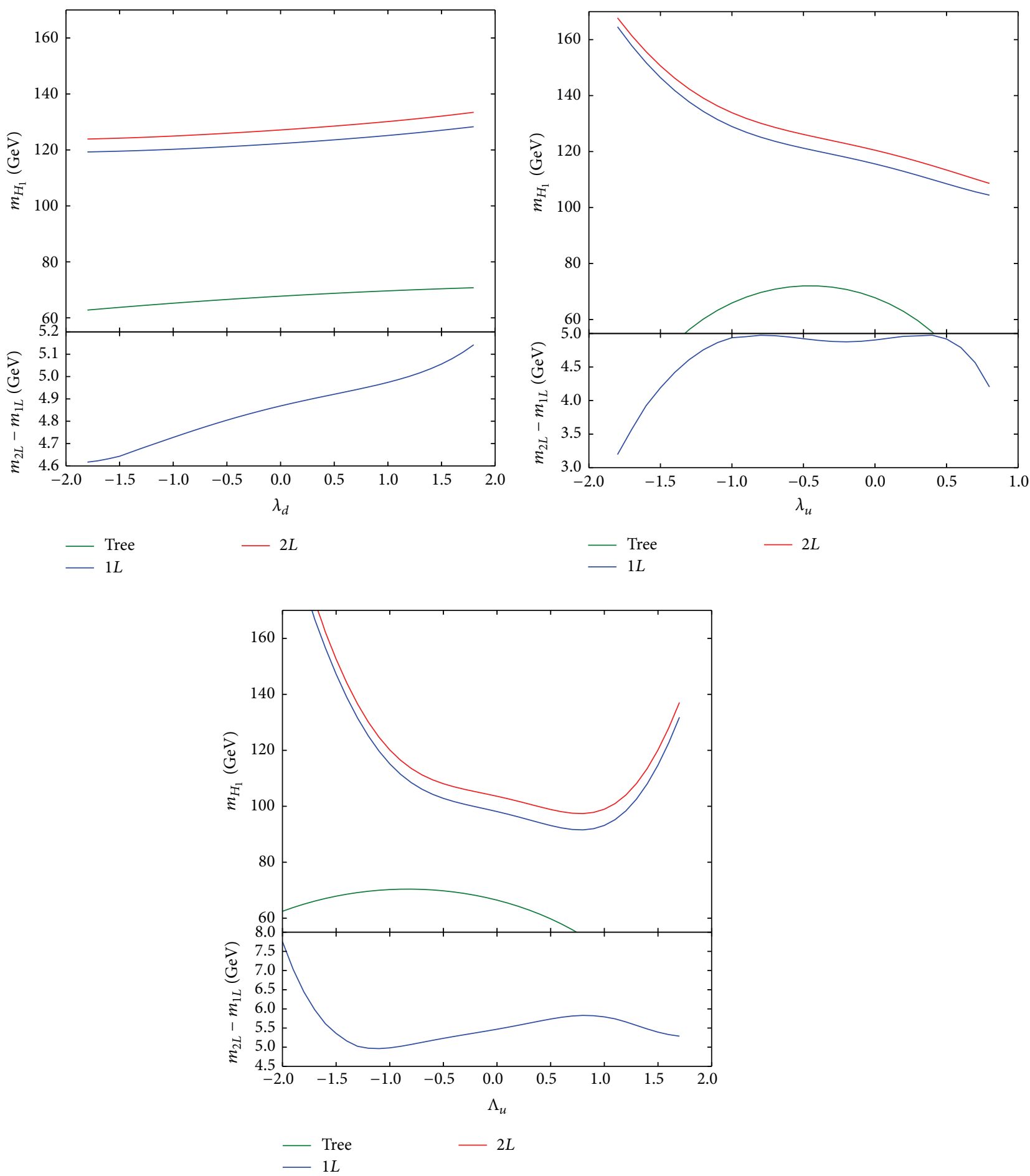

FIGURE 1: Lightest MRSSM Higgs boson mass $m_{H_{1}}$ and the difference $m_{2 L}-m_{1 L}$ between masses calculated at the two-loop and one-loop level, as a function of $\lambda_{d}, \lambda_{u}$, and $\Lambda_{u}$, respectively. In the upper parts of the figure lines from top to bottom correspond to two-loop, one-loop, and tree-level calculations. All other parameters are set to the values of benchmark point BMP1 with $\tan \beta=3$ (see Table 2).

close to the experimental one. In the preferred parameter regions, $\lambda, \Lambda$ are large but still moderate enough not to blow up the two-loop contributions. Although the large values of $\Lambda$ couplings will lead to Landau poles at scales below the GUT scale; this is not problem for our phenomenological approach, and even in top-down approaches as in [40] perturbativity up to the GUT scale is not required.

Overall, the total two-loop contributions (including the ones to be discussed in the subsequent section) are in the range between 4 and $5 \mathrm{GeV}$, except in the very large $\lambda, \Lambda$ regions. This is in agreement with the estimate given in [17], 

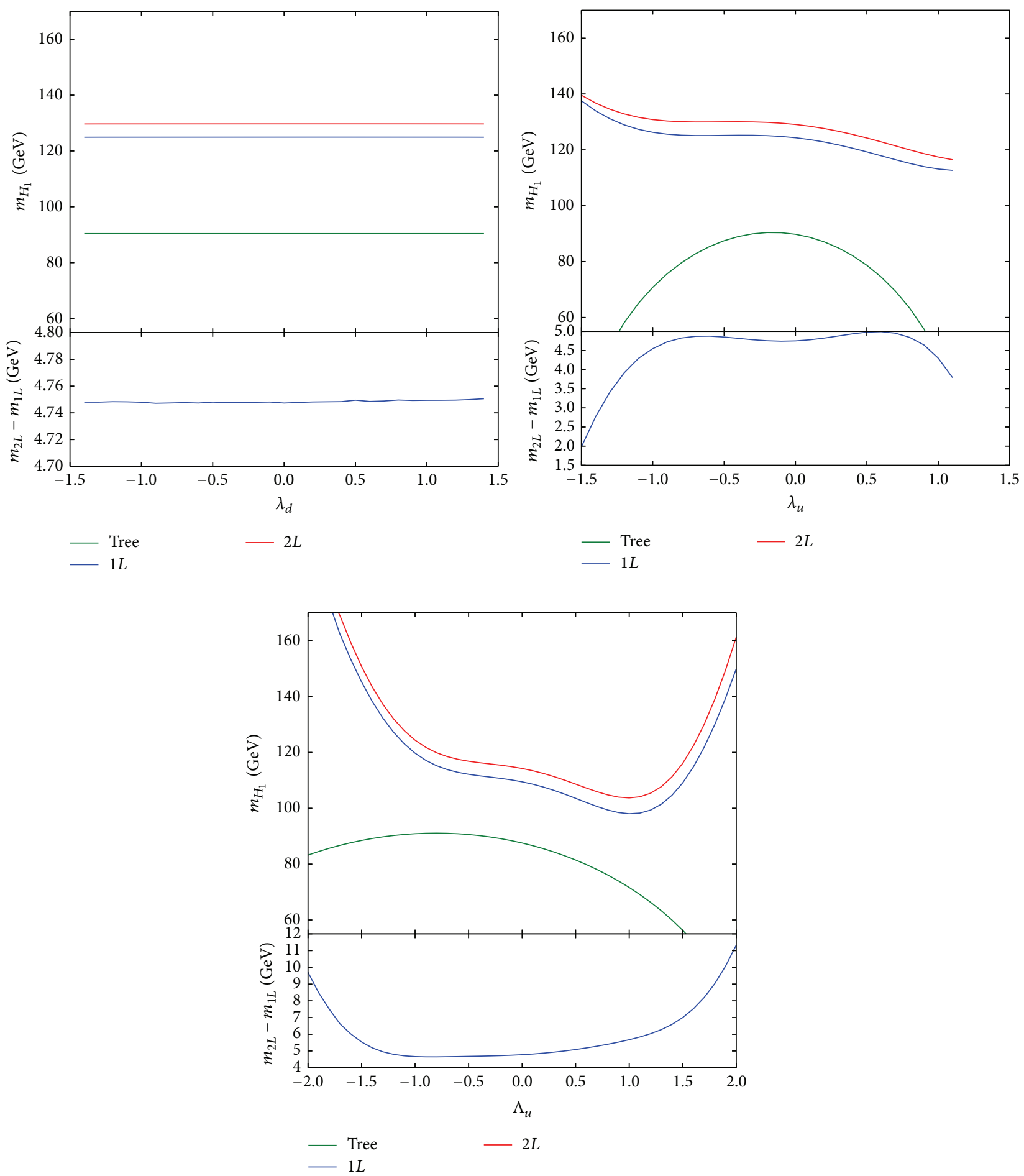

FIgURE 2: As in Figure 1, but for benchmark point BMP3 with $\tan \beta=40$ (see Table 2).

and it confirms the validity of the perturbative expansion in spite of the large one-loop corrections.

\section{QCD Corrections and the Two-Loop Corrected Higgs Boson Mass}

At two-loop level the strongly interacting sector and the strong coupling $\alpha_{s}$ appear directly in the Higgs boson mass predictions. These two-loop corrections involve not only the gluon but also the Dirac gluino and the sgluon, the scalar component of the octet superfield $\widehat{O}$. They can be expected to be sizable, and they depend on the gluino Dirac mass and sgluon soft mass parameters. These parameters already play a role at lower order, appearing in corrections to $Y_{t}$ (through threshold corrections to $\widehat{\alpha}_{s}$ ), though the influence on, for 

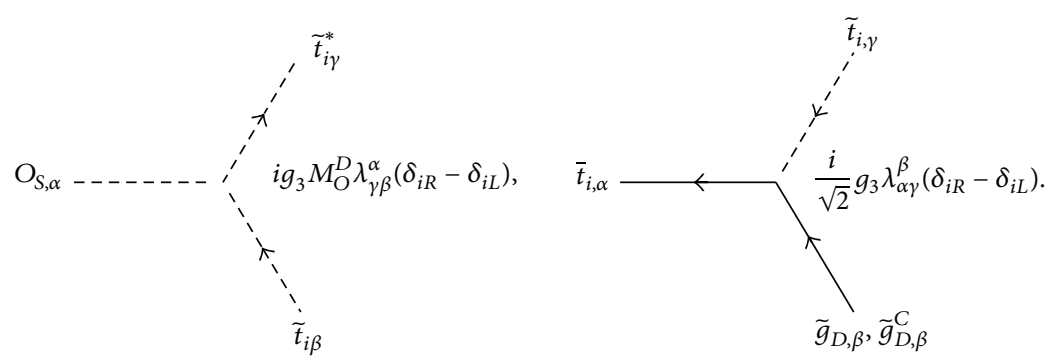

FIGURE 3: Feynman rules needed to evaluate diagrams of Figure 4. In the right diagram, the charge-conjugated gluino $\widetilde{g}_{D, \beta}^{C}$ applies in the case of $i=L, \widetilde{g}_{D, \beta}$ in the case of $i=R$.
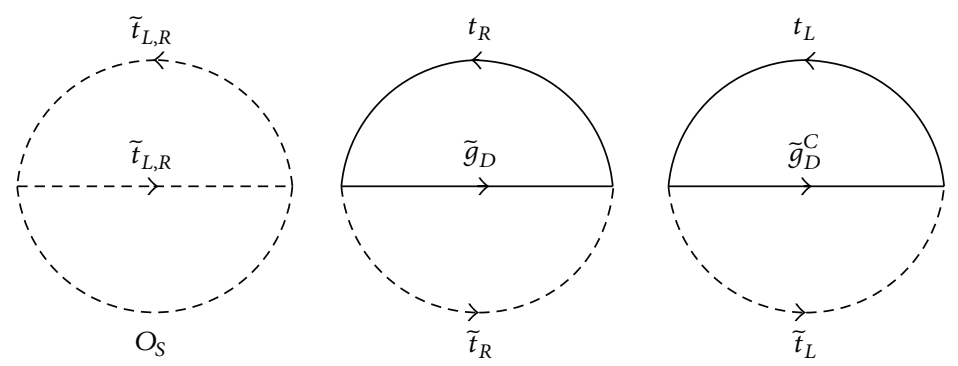

FIGURE 4: Two-loop diagrams contributing to the Higgs boson mass via (6) which depend on the Dirac mass $M_{O}^{D}$ and the soft sgluon mass $m_{\mathrm{O}}$. We only draw diagrams involving top/stop; similar diagrams exist for all quark/squark flavors.

example, $\overline{\mathrm{DR}}$ top mass is negligible. The gluino Dirac mass parameter $M_{O}^{D}$ appears not only directly as the gluino mass but via (2), also in couplings and mass terms of sgluons, inducing the mass splitting. In [17] a simplifying assumption was made that masses of the scalar and pseudoscalar components of (complex) sgluon field were equal, since it was unimportant for that analysis between the real and imaginary parts of the sgluon field, $O=(1 / \sqrt{2})\left(O_{S}+i O_{A}\right)$. The masses of the scalar sgluons $O_{S}$ and pseudoscalar sgluons $O_{A}$ are related by the tree-level formula $m_{\mathrm{O}_{S}}^{2}=4\left(M_{O}^{D}\right)^{2}+m_{\mathrm{O}_{A}}^{2}$, where $m_{\mathrm{O}_{A}}^{2}$ is equal to the soft-breaking parameter $m_{O}^{2}[26,28]$. The relevant vertices and Feynman rules are depicted in Figure 3. We assume real $M_{O}^{D}$, so only the scalar $O_{S}$ acquires the direct coupling to sfermions proportional to $M_{O}^{D}$, via (2).

The structure of the strong corrections is thus markedly different from the MSSM case, where only the Majorana gluino and the gluon appear. In the following, we study the magnitude and the behavior of the corrections as a function of the parameters $M_{O}^{D}$ and $m_{O}^{2}$.

4.1. Analytic Formulas. As in the previous section, we begin with an analytic approximation for the leading contributions of $\mathcal{O}\left(\alpha_{t} \alpha_{s}\right)$, that is, two-loop strong corrections proportional to $Y_{t}^{2}$. This provides us with qualitative insight and serves as a check of the code. Generally, in the gaugeless limit (in which the two electroweak gauge couplings $g_{1,2}$ are neglected), the two-loop corrections from gluinos and sgluons contribute only to the diagonal part of $\left\{\phi_{d}, \phi_{u}\right\}$ submatrix of the scalar Higgs boson mass matrix. In the MRSSM $\mathcal{O}\left(\alpha_{t} \alpha_{s}\right)$ terms contribute only to $\phi_{u} \phi_{u}$ element. This already constitutes a difference to the MSSM, where $\mu$-term violates $R$-symmetry and Peccei-Quinn symmetry leading to couplings of stops to $\phi_{d}$.

Figure 4 shows two-loop diagrams contributing to the Higgs boson mass at $\mathcal{O}\left(\alpha_{t} \alpha_{s}\right)$ that explicitly depend on $m_{O}$ and/or $M_{O}^{D}$. These diagrams provide the following contribution to the effective potential:

$$
\begin{aligned}
V_{\mathrm{eff}}^{(2)}= & \frac{8 g_{3}^{2}}{\left(16 \pi^{2}\right)^{2}}\left(M_{O}^{D}\right)^{2} \sum_{i=L, R} f_{S S S}\left(m_{\tilde{t}_{i}}^{2}, m_{\tilde{t}_{i}}^{2}, m_{O_{S}}^{2}\right) \\
& +\frac{8 g_{3}^{2}}{\left(16 \pi^{2}\right)^{2}} \sum_{i=L, R} f_{F F S}\left(m_{t}^{2}, m_{\tilde{t}_{i}}^{2}, m_{\tilde{g}_{D}}^{2}\right),
\end{aligned}
$$

where the functions $f_{S S S}$ and $f_{F F S}$ are defined in [42]. The effective potential $V_{\text {eff }}^{(2)}$ depends on $v_{u}$ through stop masses, which in the gaugeless limit approach

$$
\begin{aligned}
& m_{\tilde{t}_{L} \tilde{t}_{L}}^{2} \longrightarrow m_{q}^{2}+\frac{1}{2} Y_{t}^{2} v_{u}^{2}, \\
& m_{\tilde{t}_{R} \tilde{t}_{R}}^{2} \longrightarrow m_{u}^{2}+\frac{1}{2} Y_{t}^{2} v_{u}^{2} .
\end{aligned}
$$

Equation (6) can be obtained from [42] by applying translation rules from real fields to complex ones. Many such rules can be found in [36]; an additional rule needed here for the case of a Lagrangian $\mathscr{L} \ni-c \Phi_{1}\left|\Phi_{2}\right|^{2}$, where $\Phi_{1}, c \in \mathbb{R}, \Phi_{2} \in$ $\mathbb{C}$, is $V_{S S S}=(1 / 2)|c|^{2} f_{S S S}\left(m_{1}^{2}, m_{2}^{2}, m_{2}^{2}\right)$.

An important difference to the MSSM is that contributions with fermion mass insertions, corresponding to $\overline{F F} S$ type contributions in [42], are not present in the MRSSM. Such contributions vanish due to the lack of $L-R$ mixing between squarks. Hence the gluino mass appears in a simpler 
way than in the MSSM. Likewise, the sgluon only enters via the SSS-type diagram of Figure 4. An SS-type diagram vanishes due to the color structure.

The corresponding two-loop contribution to $\phi_{u} \phi_{u}$ Higgs boson mass matrix element in zero-momentum approximation is then given by (as pointed out in [36], in SARAH and SPheno the two-loop tadpole contributions are included directly in vacuum minimization condition and not in (8))

$$
\left[\Delta m_{H_{1}}^{2}\right]_{\phi_{u} \phi_{u}}=\left(\frac{\partial^{2}}{\partial v_{u} \partial v_{u}}-\frac{1}{v_{u}} \frac{\partial}{\partial v_{u}}\right) V_{\mathrm{eff}}^{(2)} .
$$

For large $\tan \beta$, corrections of order $\mathcal{O}\left(\alpha_{b} \alpha_{s}\right)$ cannot be neglected any more. But since they contribute only to $\phi_{d} \phi_{d}$ matrix element, their impact on mass of the lightest Higgs, which stems mainly from $\phi_{u} \phi_{u}$ element, is small. Results of (6) were compared with the results of two-loop routines from the SARAH-generated SPheno module.

4.2. Numerical Analysis. We now turn to the numerical analysis of the complete two-loop corrections to the SM-like Higgs boson mass, using the full evaluation within the framework of SARAH and SPheno. Figures 5(a) and 5(b) focus on the gluino and sgluon mass dependence, which arises mainly from the $\mathcal{O}\left(\alpha_{t} \alpha_{s}\right)$ corrections; they show the two-loop corrections as a function of the gluino mass parameter for two different values of the soft sgluon mass, $m_{O}=2$ and $10 \mathrm{TeV}$ for two benchmarks BMP1 and BMP3; other parameters are fixed at benchmark values. For comparison, the two-loop result without the sgluon contribution is shown as well (i.e., without the first diagram of Figure 4). We also plot the MSSM prediction with strong stop mixing and without any sfermion mixing at tree-level.

Figures 5(a) and 5(b) show that the dependence in the MRSSM without sgluon contributions is very similar to the one in the MSSM without stop mixing. The corresponding thin solid red and thin dashed light blue curves in Figure 5 show a characteristic drop for large gluino masses. This is understandable as in the MSSM without sfermion mixing the gluino contribution is precisely the same as in the MRSSM and given by the two corresponding diagrams in Figure 4 . The Dirac or Majorana nature of the gluino does not matter since the Dirac partner, the octet superfield $\widehat{O}$, has no direct couplings to quark superfields. A few $\mathrm{TeV}$ gluino masses slightly increase the Higgs boson mass, but for larger values of $M_{O}^{D}$ the $f_{F F S}$ function becomes negative and drives the correction downwards.

In the full MRSSM calculations, including the sgluon diagrams strongly changes the behavior. Surprisingly, the full MRSSM two-loop contributions resemble the MSSM contributions with large stop mixing. In both cases, large gluino masses strongly enhance the Higgs boson mass, however, for different reasons. In the MSSM the increase can be traced back to the additional $\overline{F F}$-type diagram which is directly proportional to $M_{O}^{D}$ and which vanishes in the limit of no stop mixing. In the MRSSM, on the other hand, the sgluon diagram grows with $M_{O}^{D}$ due to both the sgluon-stopstop coupling, which scales like $M_{O}^{D}$, and an increase in the scalar (but not pseudoscalar) sgluon mass. Due to the sgluon contributions the total two-loop contributions to the Higgs boson mass in the MRSSM are larger than the ones in the MSSM. They are further increased by heavy sgluons.

Figure 5(c) compares the numerical impact of individual contributions by successively switching off contributions. It allows us to read off the contributions from sgluon, gluino, and gluon, of $\mathcal{O}\left(\alpha_{t}^{2}, \alpha_{t} \alpha_{b}\right)$, and the remaining two-loop contributions (particularly $\lambda, \Lambda$ contributions). The gluon diagrams alone contribute approximately $+4 \mathrm{GeV}$. The negative gluino and the positive sgluon corrections together amount to an additional upward shift of the Higgs boson mass, which can reach several $\mathrm{GeV}$ for large Dirac gluino masses. The remaining contributions are far smaller and amount to around $-1 \mathrm{GeV}$ for $\mathcal{O}\left(\alpha_{t}^{2}, \alpha_{t} \alpha_{b}\right)$ contributions and $+0.5 \mathrm{GeV}$ for the remaining contributions.

\section{Update of Benchmarks}

In this section we present an update of the analysis of [17], using the more precise evaluation of the Higgs boson mass. Reference [17] studied the mass predictions of $W$ and lightest Higgs bosons in the MRSSM and showed that agreement with experimental data is possible, in spite of tree-level shifts from violations of custodial symmetry and from mixing with other Higgs states, respectively.

Table 2 shows benchmark parameter points defined in that reference. They exemplify parameter regions in which $m_{W}$ and $m_{H_{1}}$ agree with experiment. They are characterized by large $|\Lambda| \approx 1$, rather light Dirac higgsinos and gauginos, and they have $\tan \beta=3,10,40$, respectively.

For all three benchmark points the two-loop correction to $m_{H_{1}}$ is around $+5 \mathrm{GeV}$. As discussed in the previous sections, the largest part of this is due to $\mathcal{O}\left(\alpha_{t} \alpha_{s}\right)$ corrections. The MRSSM-specific corrections of $\mathcal{O}\left(\alpha_{\Lambda}^{2}\right)$ are small since the values of $\Lambda_{u}$, though large, are still not as large as needed to make these corrections dominate; see Figures 1 and 2 for two out of three benchmarks. The magnitude of the total two-loop correction is consistent with the theory error estimate given in [17].

The upward shift of $m_{H_{1}}$ implies that it is easier to obtain agreement with the measured value; that is, smaller values of $\left|\Lambda_{u}\right|$ are sufficient. In Table 3 we provide new, slightly modified benchmark points, whose definitions differ only in the values of $\Lambda_{u}$. The two-loop Higgs boson mass prediction agrees well with experiment, and the good agreement of $m_{W}$ with experiment is unchanged. Likewise, both the old and the new set of benchmark points pass checks against HiggsBounds [43-45] and HiggsSignals $[46,47]$.

In Figure 6 we give an update to some of the subfigures from Figures 4 and 5 of [17]. These show the predictions of $m_{W}$ and $m_{H_{1}}$ as contour lines in several two-dimensional parameter spaces. The Higgs boson mass is evaluated at the two-loop level. As discussed before, with the exception of the regions of very large $\Lambda$, there is a general positive contribution to the lightest Higgs boson mass between 4 and $5 \mathrm{GeV}$. Accordingly, the contour lines, in particular the central green region in which the Higgs boson mass agrees 


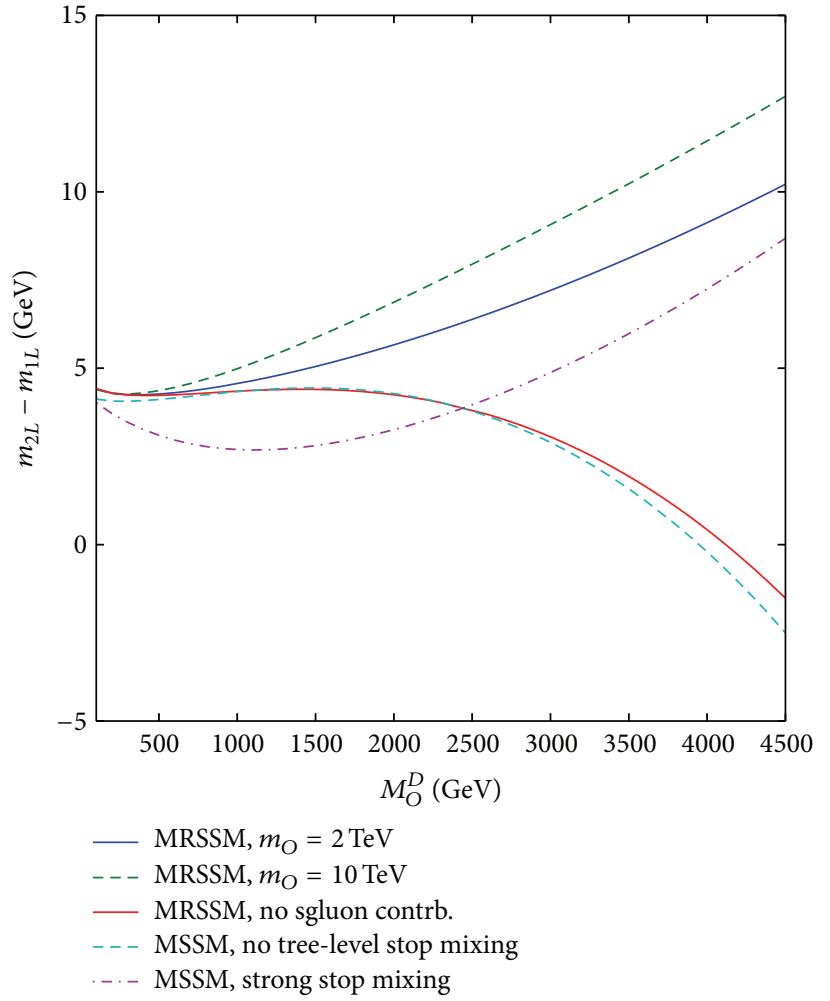

(a)

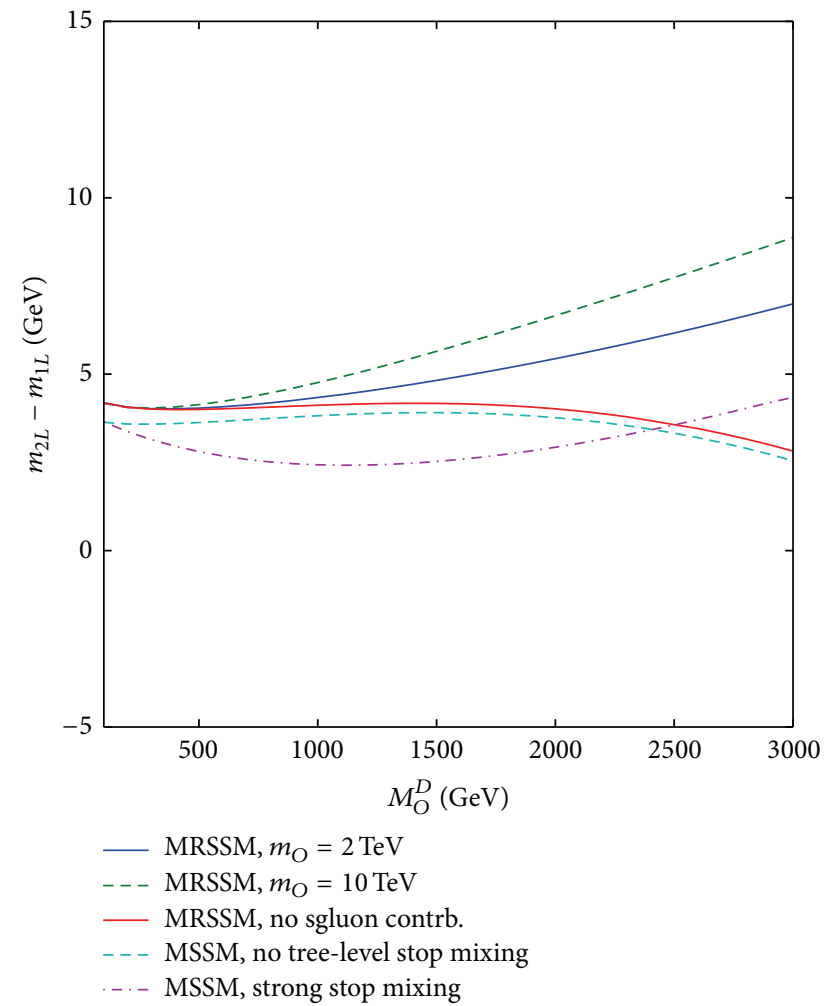

(b)

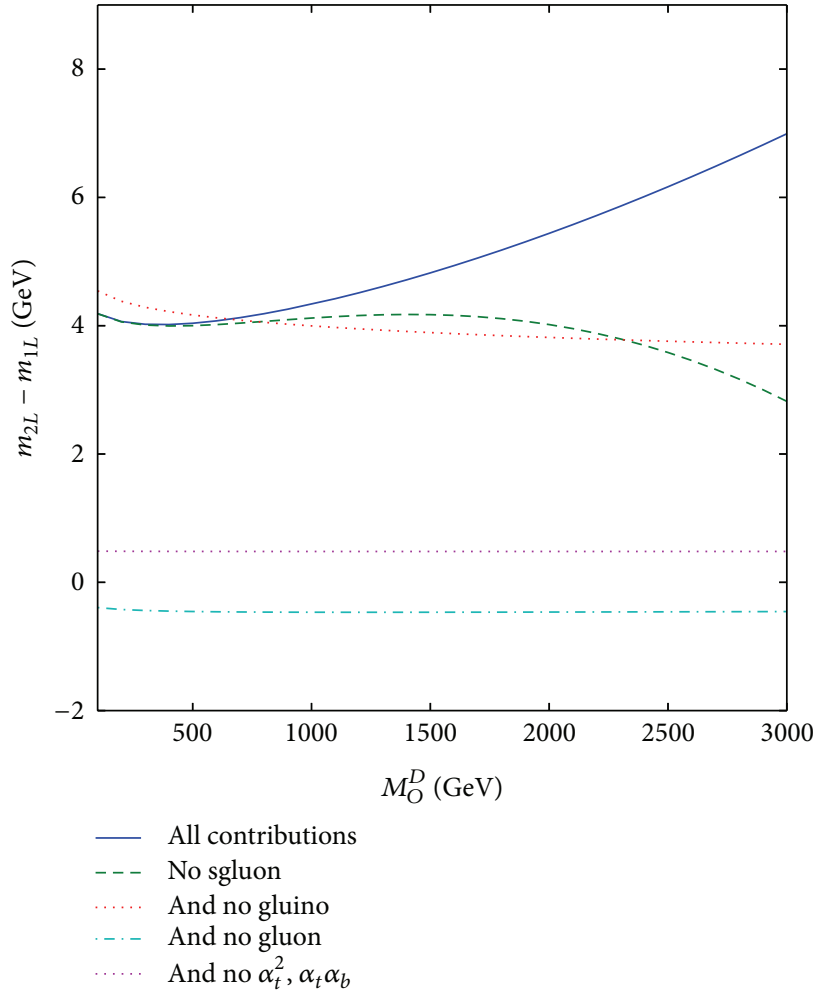

(c)

Figure 5: Two-loop contributions to the SM-like Higgs boson mass depending on the gluino mass in the MRSSM for BMP1 (a) and BMP3 (b) and two different values of the soft sgluon mass parameter $m_{\mathrm{O}}=2 \mathrm{TeV}$ (thick solid blue line) and $10 \mathrm{TeV}$ (thick dashed green line) with all contributions, respectively, and without the sgluon contributions (thin solid red line). For comparison also the MSSM contributions for no (thin dashed light blue line) and maximal (purple dotted line) stop mixing are plotted. The chosen MSSM parameters are given in Table 1. For BMP3 and $m_{\mathrm{O}}=2 \mathrm{TeV}$ (c) shows the result, when successively switching off dominating and subdominating contributions. 
TABLE 1: Definition of the fixed parameters for the MSSM points in Figures 5(a), 5(b) and 5(c). All parameters in $\mathrm{GeV}^{\text {or }} \mathrm{GeV}^{2}$, where appropriate. The stop mixing parameter $X_{t}$ is given for both cases of no and large stop mixing.

\begin{tabular}{ccccccccccc}
\hline & $\tan \beta$ & $M_{1}$ & $M_{2}$ & $\mu$ & $m_{A}$ & $m_{q, u, d ;(3,3)}^{2}$ & $m_{q, u, d}^{2}$ & $m_{l, e}^{2}$ & $A_{\tau, b}$ & $X_{t}$ \\
\hline BMP1 & 3 & 600 & 500 & 400 & 700 & $1000^{2}$ & $2000^{2}$ & $1000^{2}$ & 0 & $0 / 2000$ \\
BMP3 & 40 & 250 & 500 & 400 & 700 & $1000^{2}$ & $2000^{2}$ & $1000^{2}$ & 0 & $0 / 2000$ \\
\hline
\end{tabular}

TABLE 2: Benchmark points of [17]. Dimensionful parameters are given in $\mathrm{GeV}$ or $\mathrm{GeV}^{2}$, as appropriate. The first two parts define input parameters. The third part shows parameters derived from electroweak symmetry breaking after solving the tadpole equations at two loops. The last part gives the theory predictions for the Higgs boson mass at the two-loop level and further quantities relevant to comparison with experiment.

\begin{tabular}{|c|c|c|c|}
\hline & BMP1 & BMP2 & BMP3 \\
\hline $\tan \beta$ & 3 & 10 & 40 \\
\hline$B_{\mu}$ & $500^{2}$ & $300^{2}$ & $200^{2}$ \\
\hline$\lambda_{d}, \lambda_{u}$ & $1.0,-0.8$ & $1.1,-1.1$ & $0.15,-0.15$ \\
\hline$\Lambda_{d}, \Lambda_{u}$ & $-1.0,-1.2$ & $-1.0,-1.0$ & $-1.0,-1.15$ \\
\hline$M_{B}^{D}$ & 600 & 1000 & 250 \\
\hline$m_{R_{u}}^{2}$ & $2000^{2}$ & $1000^{2}$ & $1000^{2}$ \\
\hline$\mu_{d}, \mu_{u}$ & \multicolumn{3}{|c|}{400,400} \\
\hline$M_{W}^{D}$ & \multicolumn{3}{|c|}{500} \\
\hline$M_{O}^{D}$ & \multicolumn{3}{|c|}{1500} \\
\hline$m_{T}^{2}, m_{S}^{2}, m_{O}^{2}$ & \multicolumn{3}{|c|}{$3000^{2}, 2000^{2}, 1000^{2}$} \\
\hline$m_{\mathrm{Q} ; 1,2}^{2}, m_{\mathrm{Q} ; 3}^{2}$ & \multicolumn{3}{|c|}{$2500^{2}, 1000^{2}$} \\
\hline$m_{D ; 1,2}^{2}, m_{D ; 3}^{2}$ & \multicolumn{3}{|c|}{$2500^{2}, 1000^{2}$} \\
\hline$m_{U ; 1,2}^{2}, m_{U ; 3}^{2}$ & \multicolumn{3}{|c|}{$2500^{2}, 1000^{2}$} \\
\hline$m_{L}^{2}, m_{E}^{2}$ & \multicolumn{3}{|c|}{$1000^{2}$} \\
\hline$m_{R_{d}}^{2}$ & \multicolumn{3}{|c|}{$700^{2}$} \\
\hline$v_{S}$ & 4.96 & 0.67 & -0.30 \\
\hline$v_{T}$ & -0.34 & -0.20 & -0.34 \\
\hline$m_{H_{d}}^{2}$ & $673^{2}$ & $743^{2}$ & $1160^{2}$ \\
\hline$m_{H_{u}}^{2}$ & $-535^{2}$ & $-542^{2}$ & $-541^{2}$ \\
\hline$m_{H_{1}}$ & $130.3 \mathrm{GeV}$ & $130.3 \mathrm{GeV}$ & $129.8 \mathrm{GeV}$ \\
\hline$m_{W}$ & $80.400 \mathrm{GeV}$ & $80.384 \mathrm{GeV}$ & $80.393 \mathrm{GeV}$ \\
\hline HiggsBounds's obsratio & 0.67 & 0.68 & 0.67 \\
\hline HiggsSignals' $p$ value & 0.03 & 0.03 & 0.03 \\
\hline
\end{tabular}

with experiment, shift to slightly lower values of $\Lambda$. Also, the overlap region, where Higgs and $W$ boson masses agree with experiment, is enlarged.

\section{Conclusions}

In this work we have presented the impact of two-loop corrections on the mass of the lightest Higgs boson in the MRSSM. The calculation has been performed using the framework of SARAH in the approximation of the vanishing electroweak gauge couplings and external momenta of the Higgs self-energies. The code has been cross-checked with an analytic calculation of the most important new corrections. We have separately analyzed the impact of contributions
TABLE 3: Adapted benchmark points; other parameters are as given in Table 2.

\begin{tabular}{lccc}
\hline & BMP1 $^{\prime}$ & BMP2 $^{\prime}$ & BMP3 $^{\prime}$ \\
\hline$\Lambda_{u}$ & -1.11 & -0.85 & -1.03 \\
\hline$v_{S}$ & 5.2 & 1.01 & -0.22 \\
$v_{T}$ & -0.25 & -0.02 & -0.21 \\
$m_{H_{d}}^{2}$ & $674^{2}$ & $764^{2}$ & $1160^{2}$ \\
$m_{H_{u}}^{2}$ & $-502^{2}$ & $-512^{2}$ & $-516^{2}$ \\
\hline$m_{H_{1}}$ & $125.3 \mathrm{GeV}$ & $125.5 \mathrm{GeV}$ & $125.4 \mathrm{GeV}$ \\
$m_{W}$ & $80.397 \mathrm{GeV}$ & $80.381 \mathrm{GeV}$ & $80.386 \mathrm{GeV}$ \\
HiggsBounds's obsratio & 0.61 & 0.65 & 0.87 \\
HiggsSignals' $p$ value & 0.72 & 0.66 & 0.72 \\
\hline
\end{tabular}

involving $\lambda, \Lambda$-couplings, which already appear in the oneloop corrections, and of the strong corrections involving gluon, Dirac gluino, and sgluon exchange.

In the previous work [17] and the present paper we have found that the lightest Higgs boson mass in the MRSSM differs from the one in the usual MSSM in several respects. At tree-level the additional mixing with additional scalar states reduces the MRSSM Higgs mass below the MSSM value. At the one-loop level, the top/stop contributions cannot be as large as in the MSSM, because stop mixing is forbidden by $R$-symmetry. However, the new contributions from the superpotential $\lambda, \Lambda$-terms have a similar structure as the top/stop contributions. If $\lambda, \Lambda$-couplings are similar in magnitude to the top Yukawa coupling, the lightest Higgs boson mass can easily be in the ballpark of the experimentally allowed range.

The two-loop corrections governed by these $\lambda, \Lambda$ couplings, however, amount to only $1 \mathrm{GeV}$ or less in parameter regions in which the Higgs boson mass agrees with experiment. The most important two-loop contributions are the strong corrections of $\mathcal{O}\left(\alpha_{t} \alpha_{s}\right)$. As we have shown the Dirac gluino and gluon contributions alone are very similar to the MSSM strong contributions for vanishing stop mixing. The inclusion of the sgluons changes the picture. The sgluon contributions are positive and rise with the Dirac gluino mass, such that the total $\mathcal{O}\left(\alpha_{t} \alpha_{s}\right)$ corrections of the MRSSM are larger than the ones of the MSSM, independently of the magnitude of stop mixing.

Overall, the MRSSM two-loop corrections to the lightest Higgs boson mass are typically positive. For example, for the benchmark parameter points proposed in [17], the twoloop corrections to the Higgs boson mass amount to approximately $+5 \mathrm{GeV}$, within the error estimate of that reference. Since perturbation theory shows a converging behavior and since $\lambda, \Lambda$-corrections are subdominant (for $|\lambda|,|\Lambda|$ less than 


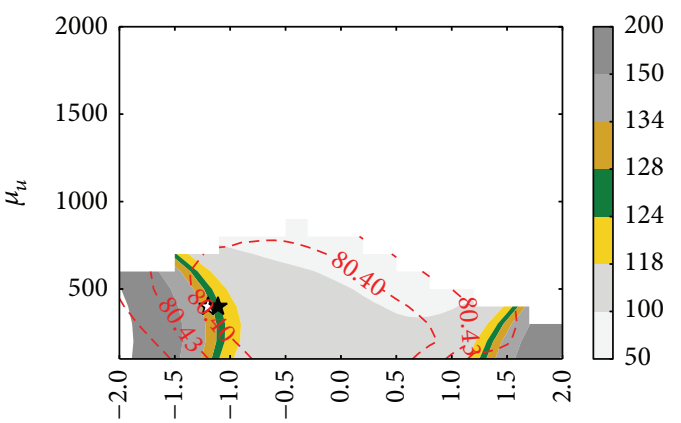

$\Lambda_{u}$

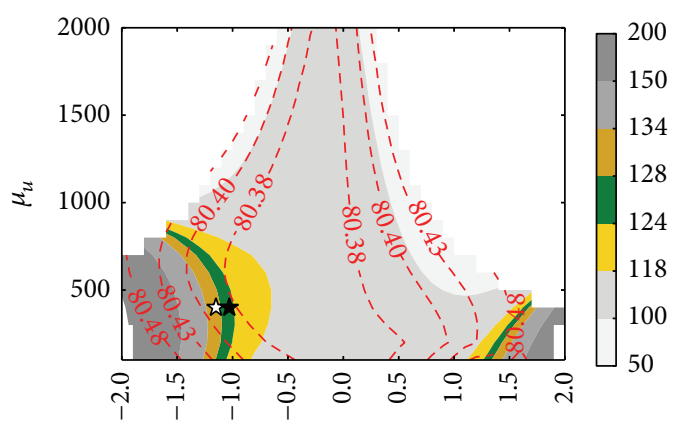

$\Lambda_{u}$

(a)

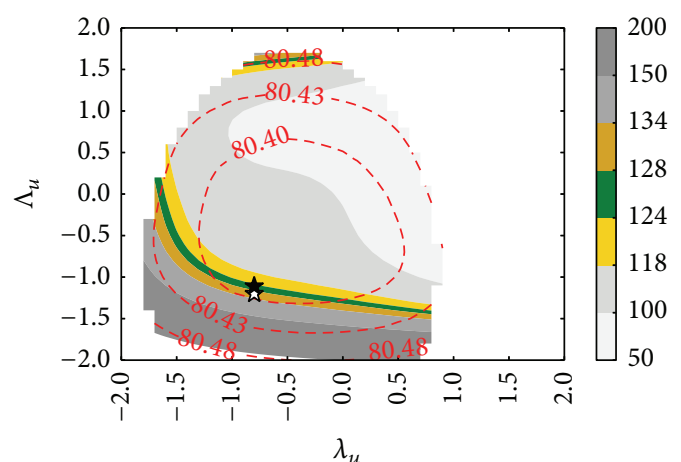

$\lambda_{u}$

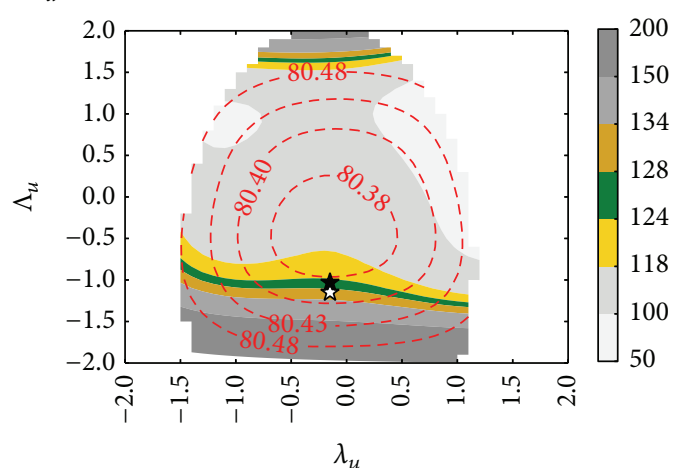

(b)

FIGURE 6: Contour plots showing the behavior of $m_{H_{1}}$ given by the color map and $m_{W}$ by the red contour lines: (a) for BMP1, (b) for BMP3, for different combinations of model parameters. The white stars mark the original benchmark points from [17], whereas the black ones show the adapted points after taking into account the two-loop corrections.

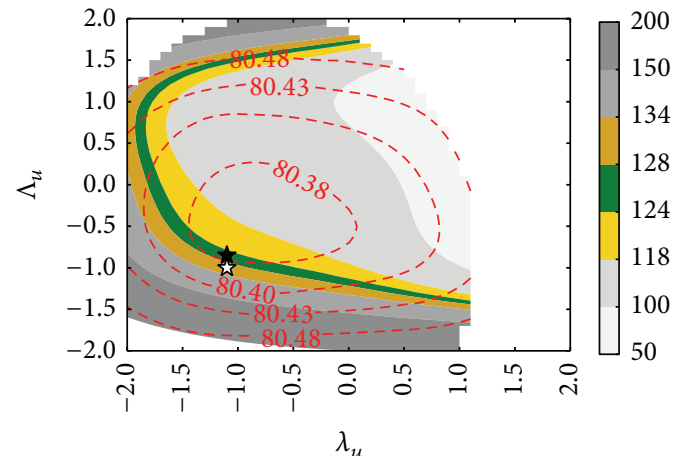

128

24

118

100 
around 1.2), we estimate the remaining theory uncertainty to be not larger than the one of the MSSM.

The positive two-loop corrections make it easier to achieve agreement between the theory prediction for the lightest Higgs boson mass and the measured value. We have provided an update of the analysis of [17], showing parameter regions of simultaneous agreement of the Higgs and $W$ boson mass predictions with experiment. Compared to [17], the allowed parameter regions are slightly larger and located at smaller values of $\lambda, \Lambda$-couplings.

\section{Conflict of Interests}

The authors declare that there is no conflict of interests regarding the publication of this paper.

\section{Acknowledgments}

The authors would like to thank Kilian Nickel and Florian Staub for communication about SARAH. This work is supported in part by the Polish National Science Centre Grants under OPUS-2012/05/B/ST2/03306, DEC-2012/05/B/ ST2/02597, the European Commission through the Contract PITN-GA-2012-316704 (HIGGSTOOLS), the German DAAD PPP Poland Project 56269947 "Dark Matter at Colliders," and DFG Research Training Group 1504 and the DFG Grant STO 876/4-1.

\section{References}

[1] G. Aad, B. Abbott, J. Abdallah et al., "Combined measurement of the Higgs boson mass in $p p$ collisions at $\sqrt{s}=7$ and $8 \mathrm{TeV}$ with the ATLAS and CMS experiments," Physical Review Letters, vol. 114, Article ID 191803, 2015.

[2] R. Harlander, P. Kant, L. Mihaila, and M. Steinhauser, "Higgs boson mass in supersymmetry to three loops," Physical Review Letters, vol. 100, no. 19, Article ID 191602, 4 pages, 2008.

[3] P. Kant, R. V. Harlander, L. Mihaila, and M. Steinhauser, "Light MSSM Higgs boson mass to three-loop accuracy," Journal of High Energy Physics, vol. 2010, article 104, 2010.

[4] T. Hahn, S. Heinemeyer, W. Hollik, H. Rzehak, and G. Weiglein, "High-precision predictions for the light $C P$-even higgs boson mass of the minimal supersymmetric standard model," Physical Review Letters, vol. 112, no. 14, Article ID 141801, 2014.

[5] P. Draper, G. Lee, and C. E. M. Wagner, "Precise estimates of the Higgs mass in heavy supersymmetry," Physical Review D-Particles, Fields, Gravitation and Cosmology, vol. 89, no. 5, Article ID 055023, 2014.

[6] G. Degrassi, S. Di Vita, and P. Slavich, "Two-loop QCD corrections to the MSSM Higgs masses beyond the effectivepotential approximation," The European Physical Journal C, vol. 75, no. 2, article 61, 2015.

[7] S. Borowka, T. Hahn, S. Heinemeyer, G. Heinrich, and W. Hollik, "Momentum-dependent two-loop QCD corrections to the neutral Higgs-boson masses in the MSSM," European Physical Journal C, vol. 74, no. 8, article 2994, 2014.

[8] W. Hollik and S. Paßehr, "Two-loop top-Yukawa-coupling corrections to the Higgs boson masses in the complex MSSM," Physics Letters B, vol. 733, pp. 144-150, 2014.
[9] W. Hollik and S. Paßehr, "Higgs boson masses and mixings in the complex MSSM with two-loop top-Yukawa-coupling corrections," Journal of High Energy Physics, vol. 2014, article 171, 2014.

[10] F. Staub, "Automatic calculation of supersymmetric renormalization group equations and loop corrections," Computer Physics Communications, vol. 182, no. 3, pp. 808-833, 2011.

[11] F. Staub, "SARAH 3.2: dirac gauginos, UFO output, and more," Computer Physics Communications, vol. 184, no. 7, pp. 17921809, 2013.

[12] F. Staub, "SARAH 4: a tool for (not only SUSY) model builders," Computer Physics Communications, vol. 185, no. 6, pp. 17731790, 2014.

[13] W. Porod, "SPheno, a program for calculating supersymmetric spectra, SUSY particle decays and SUSY particle production at $\mathrm{e}^{+} \mathrm{e}^{-}$colliders," Computer Physics Communications, vol. 153, no. 2, pp. 275-315, 2003.

[14] W. Porod and F. Staub, "SPheno 3.1: extensions including flavour, CP-phases and models beyond the MSSM," Computer Physics Communications, vol. 183, no. 11, pp. 2458-2469, 2012.

[15] P. Athron, J.-H. Park, D. Stöckinger, and A. Voigt, "FlexibleSUSY-a spectrum generator generator for supersymmetric models," Computer Physics Communications, vol. 190, pp. 139172, 2015.

[16] B. C. Allanach, "SOFTSUSY: a program for calculating supersymmetric spectra," Computer Physics Communications, vol. 143, no. 3, pp. 305-331, 2002.

[17] P. Diessner, J. Kalinowski, W. Kotlarski, and D. Stöckinger, "Higgs boson mass and electroweak observables in the MRSSM," Journal of High Energy Physics, vol. 2014, article 124, 2014.

[18] P. Fayet, "Supergauge invariant extension of the Higgs mechanism and a model for the electron and its neutrino," Nuclear Physics B, vol. 90, pp. 104-124, 1975.

[19] A. Salam and J. Strathdee, "Supersymmetry and fermionnumber conservation," Nuclear Physics B, vol. 87, no. 1, pp. 8592, 1975.

[20] G. D. Kribs, E. Poppitz, and N. Weiner, "Flavor in supersymmetry with an extended R symmetry," Physical Review D: Particles, Fields, Gravitation and Cosmology, vol. 78, no. 5, Article ID 055010, 2008.

[21] W. Buchmüller and D. Wyler, " $C P$ violation and $R$ invariance in supersymmetric models of strong and electroweak interactions," Physics Letters B, vol. 121, no. 5, pp. 321-325, 1983.

[22] E. Dudas, M. Goodsell, L. Heurtier, and P. Tziveloglou, "Flavour models with Dirac and fake gluinos," Nuclear Physics B, vol. 884, pp. 632-671, 2014.

[23] M. R. Buckley, D. Hooper, and J. Kumar, "Phenomenology of Dirac neutralino dark matter," Physical Review D, vol. 88, no. 6, Article ID 063532, 2013.

[24] E. J. Chun, J.-C. Park, and S. Scopel, "Dirac gaugino as leptophilic dark matter," Journal of Cosmology and Astroparticle Physics, vol. 2010, no. 2, article 015, 2010.

[25] G. Belanger, K. Benakli, M. Goodsell, C. Moura, and A. Pukhov, "Dark matter with Dirac and Majorana gaugino masses," Journal of Cosmology and Astroparticle Physics, vol. 2009, no. 8, article 027, 2009.

[26] P. J. Fox, A. E. Nelson, and N. Weiner, "Dirac gaugino masses and supersoft supersymmetry breaking," Journal of High Energy Physics, vol. 2002, no. 8, article 035, 2002. 
[27] T. Plehn and T. M. P. Tait, “Seeking sgluons," Journal of Physics G: Nuclear and Particle Physics, vol. 36, no. 7, Article ID 075001, 2009.

[28] S. Choi, M. Drees, J. Kalinowski, J. Kim, E. Popenda, and P. M. Zerwas, "Color-octet scalars of $\mathrm{N}=2$ supersymmetry at the LHC," Physics Letters B, vol. 672, no. 3, pp. 246-252, 2009.

[29] S. Choi, D. Choudhury, A. Freitas, J. Kalinowski, J. Kim, and P. M. Zerwas, "Dirac neutralinos and electroweak scalar bosons of $N=1 / N=2$ hybrid supersymmetry at colliders," Journal of High Energy Physics, vol. 2010, no. 8, article 025, 2010.

[30] S. Choi, D. Choudhury, A. Freitas, J. Kalinowski, and P. Zerwas, "The extended Higgs system in $R$-symmetric supersymmetry theories," Physics Letters B, vol. 697, no. 3, pp. 215-221, 2011.

[31] K. Benakli, M. D. Goodsell, and F. Staub, "Dirac gauginos and the 125 GeV Higgs," Journal of High Energy Physics, vol. 2013, no. 6, article 073, 2013.

[32] G. Aad, T. Abajyan, B. Abbott et al., "Search for pair-produced massive coloured scalars in four-jet final states with the ATLAS detector in proton-proton collisions at $\sqrt{s}=7 \mathrm{TeV}$," The European Physical Journal C, vol. 73, no. 1, article 2263, 2013.

[33] ATLAS Collaboration, "Search for anomalous production of events with same-sign dileptons and $b$ jets in $14.3 \mathrm{fb}^{-1}$ of $p p$ collisions at $\sqrt{s}=8 \mathrm{TeV}$ with the ATLAS detector," Tech. Rep. ATLAS-CONF 2013-051, 2013.

[34] W. Kotlarski, A. Kalinowski, and J. Kalinowski, "Searching for sgluons in the same-sign leptons final state at the lhc," Acta Physica Polonica B, vol. 44, no. 11, pp. 2149-2154, 2013.

[35] E. Bertuzzo, C. Frugiuele, T. Gregoire, and E. Ponton, "Dirac gauginos, R symmetry and the $125 \mathrm{GeV}$ Higgs," Journal of High Energy Physics, vol. 2015, no. 4, article 089, 2015.

[36] M. D. Goodsell, K. Nickel, and F. Staub, “Two-loop Higgs mass calculations in supersymmetric models beyond the MSSM with SARAH and SPheno," European Physical Journal C, vol. 75, no. 1, article 32, 2015.

[37] W. Hollik and D. Stöckinger, "MSSM Higgs-boson mass predictions and two-loop non-supersymmetric counterterms," Physics Letters, Section B: Nuclear, Elementary Particle and High-Energy Physics, vol. 634, no. 1, pp. 63-68, 2006.

[38] H. K. Dreiner, K. Nickel, and F. Staub, "On the two-loop corrections to the Higgs mass in trilinear R-parity violation," Physics Letters B, vol. 742, pp. 261-265, 2015.

[39] M. D. Goodsell, K. Nickel, and F. Staub, "Two-loop corrections to the Higgs masses in the NMSSM," Physical Review D, vol. 91, no. 3, Article ID 035021, 2015.

[40] S. D. L. Amigo, A. E. Blechman, P. J. Fox, and E. Poppitz, "Rsymmetric gauge mediation," Journal of High Energy Physics, vol. 2009, no. 1, article 018, 2009.

[41] K. Benakli and M. D. Goodsell, "Dirac gauginos in general gauge mediation," Nuclear Physics B, vol. 816, no. 1-2, pp. 185203, 2009.

[42] S. P. Martin, "Two-loop effective potential for a general renormalizable theory and softly broken supersymmetry," Physical Review D, vol. 65, no. 11, Article ID 116003, 2002.

[43] P. Bechtle, O. Brein, S. Heinemeyer, G. Weiglein, and K. E. Williams, "HiggsBounds: confronting arbitrary Higgs sectors with exclusion bounds from LEP and the Tevatron," Computer Physics Communications, vol. 181, no. 1, pp. 138-167, 2010.

[44] P. Bechtle, O. Brein, S. Heinemeyer, G. Weiglein, and K. E. Williams, "HiggsBounds 2.0.0: confronting neutral and charged Higgs sector predictions with exclusion bounds from LEP and the Tevatron," Computer Physics Communications, vol. 182, no. 12, pp. 2605-2631, 2011.
[45] P. Bechtle, O. Brein, S. Heinemeyer et al., "HiggsBounds-4: improved tests of extended Higgs sectors against exclusion bounds from LEP, the Tevatron and the LHC," European Physical Journal C, vol. 74, no. 3, pp. 1-32, 2014.

[46] P. Bechtle, S. Heinemeyer, O. Stål, T. Stefaniak, and G. Weiglein, "HiggsSignals: confronting arbitrary Higgs sectors with measurements at the Tevatron and the LHC," The European Physical Journal C, vol. 74, no. 2, article 2711, 2014.

[47] P. Bechtle, S. Heinemeyer, O. Stål, T. Stefaniak, and G. Weiglein, "Probing the standard model with higgs signal rates from the tevatron, the LHC and a future ILC," Journal of High Energy Physics, vol. 2014, 39 pages, 2014. 

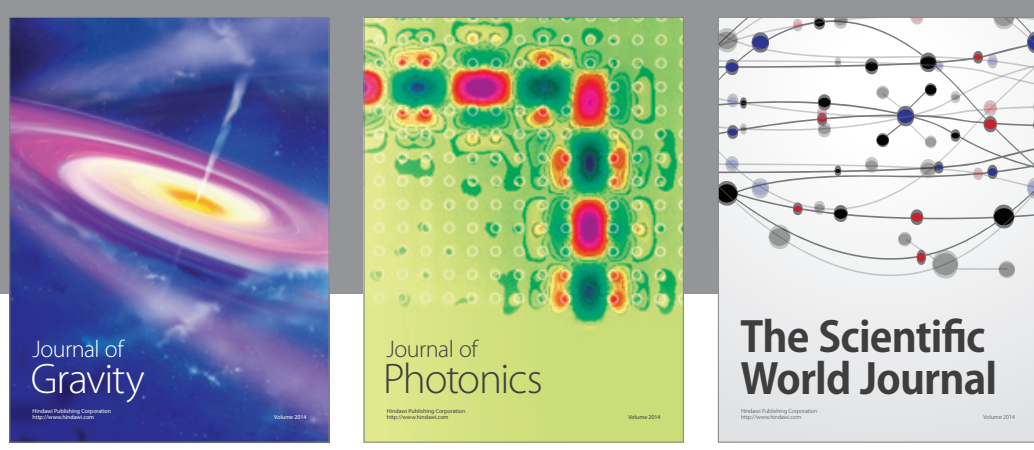

The Scientific World Journal
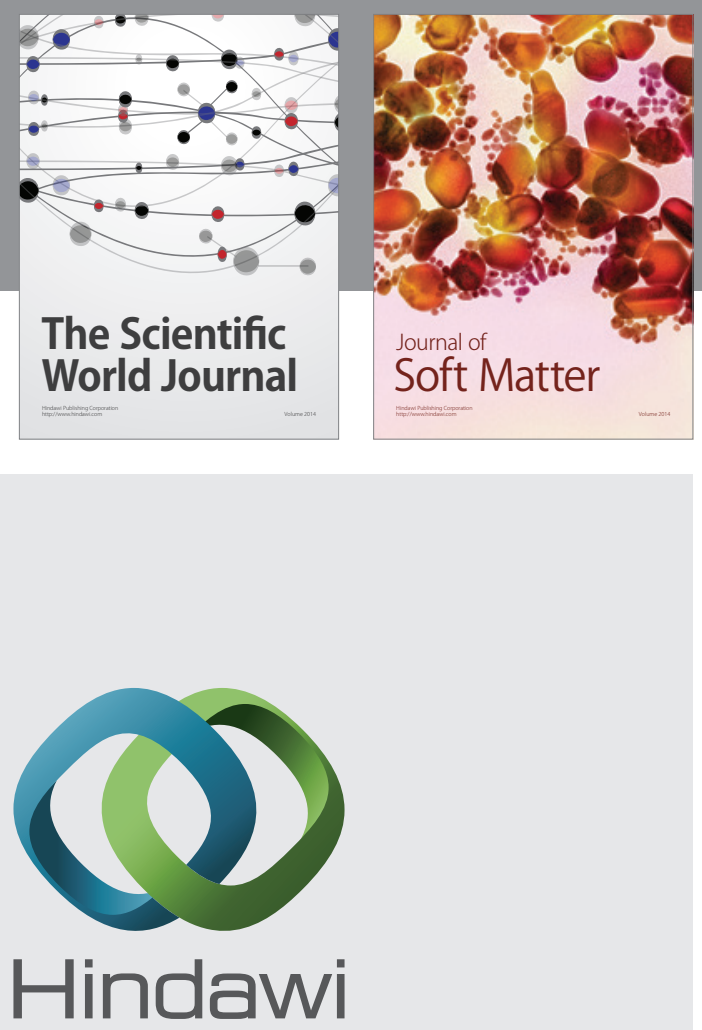

Submit your manuscripts at

http://www.hindawi.com

nternational Journal of

Statistical Mechanics
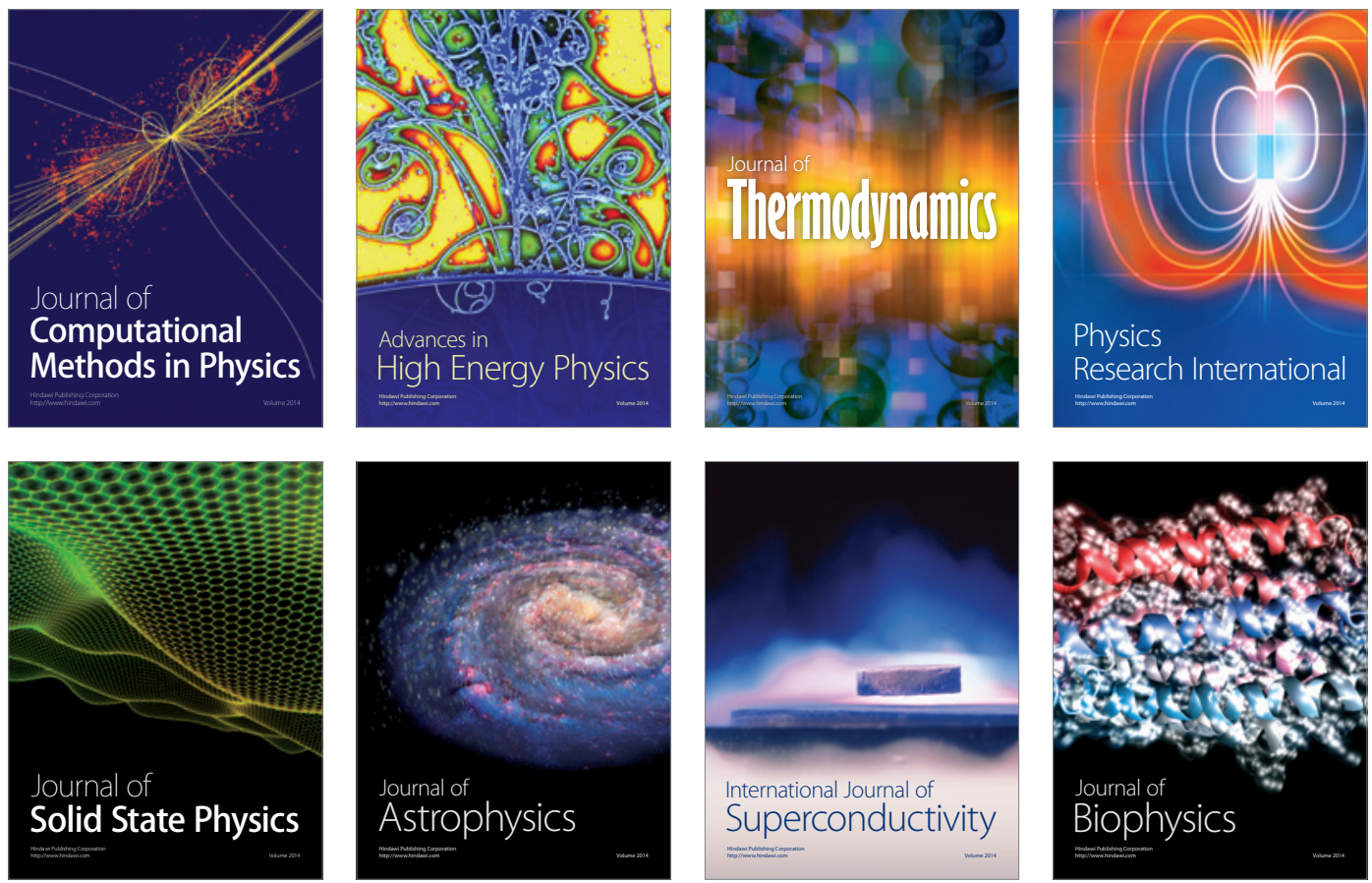
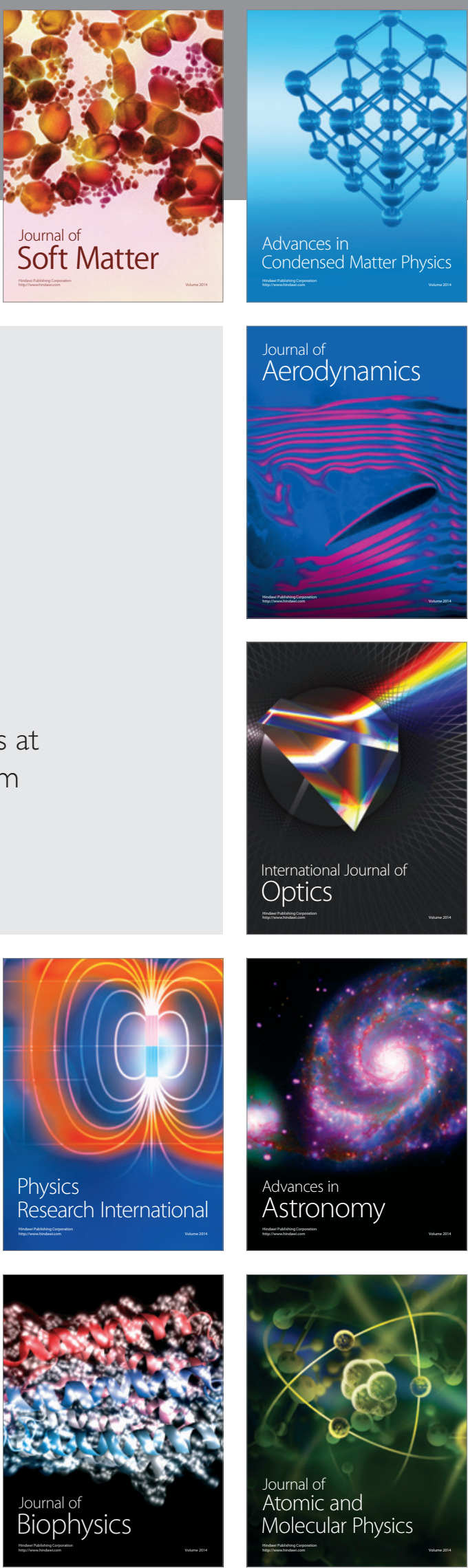